\title{
Pseudomyxoma peritonei (PMP) - a rare entity
}

\section{Zaeem I Ebrahim, MB BCh \\ Zarina I Lockhat, FFRad (D) (SA) \\ Farzanah Ismail, FCRad (D) (SA) \\ Department of Radiology, University of Pretoria}

Corresponding author: Z Ebrahim (zaeem@iafrica.com)

\section{Abstract}

Pseudomyxoma peritonei (PMP) is a rare complication of mucinous tumours of appendiceal or ovarian origin that results in peritoneal and omental implants. Clinical morbidity and mortality arise from the fact that copious amounts of extracellular and peritoneal mucin result in distortion and loss of function of visceral organs. Therapeutic paracentesis is not possible because of the nature of the mucin. Currently, new techniques are being used to attempt to debulk the mucin volume; none, however, has lead to superior outcome.

Pseudomyxoma peritonei (PMP) is a rare complication of mucinous tumours of appendiceal or ovarian origin that results in peritoneal and omental implants. In addition to the appendix and the ovary, other rare sites of apparent origin of PMP have been reported. These include the colon, stomach, gallbladder, pancreas, urachus, urinary bladder, uterine corpus, fallopian tube, breast and lung. ${ }^{1-3} \mathrm{PMP}$ is also known as 'jelly belly' or 'gelatinous ascites.'

\section{Clinical findings and complications}

While the exact pathogenesis of PMP is controversial, clinical morbidity and mortality results from the fact that copious amounts of extracellular and peritoneal mucin cause distortion and loss of function of visceral organs. ${ }^{3}$ Subsequent unrelieved compression can lead to adhesions and further morbidity, including small-bowel obstruction, renal or caval obstruction, and death. Because of the viscous, gelatinous and septated nature of the mucus, it cannot be drained by paracentesis. Impending bowel obstruction, renal compromise and discomfort may be relieved by repeated laparotomy for debridement of the mucin and subsequent decompression of the viscera.

Ronnett et al. first described a widely accepted and useful definition of PMP into three pathological subtypes with different pathological characteristics and different prognoses: disseminated peritoneal adenomucinosis (DPAM) which remains potentially non-invasive and

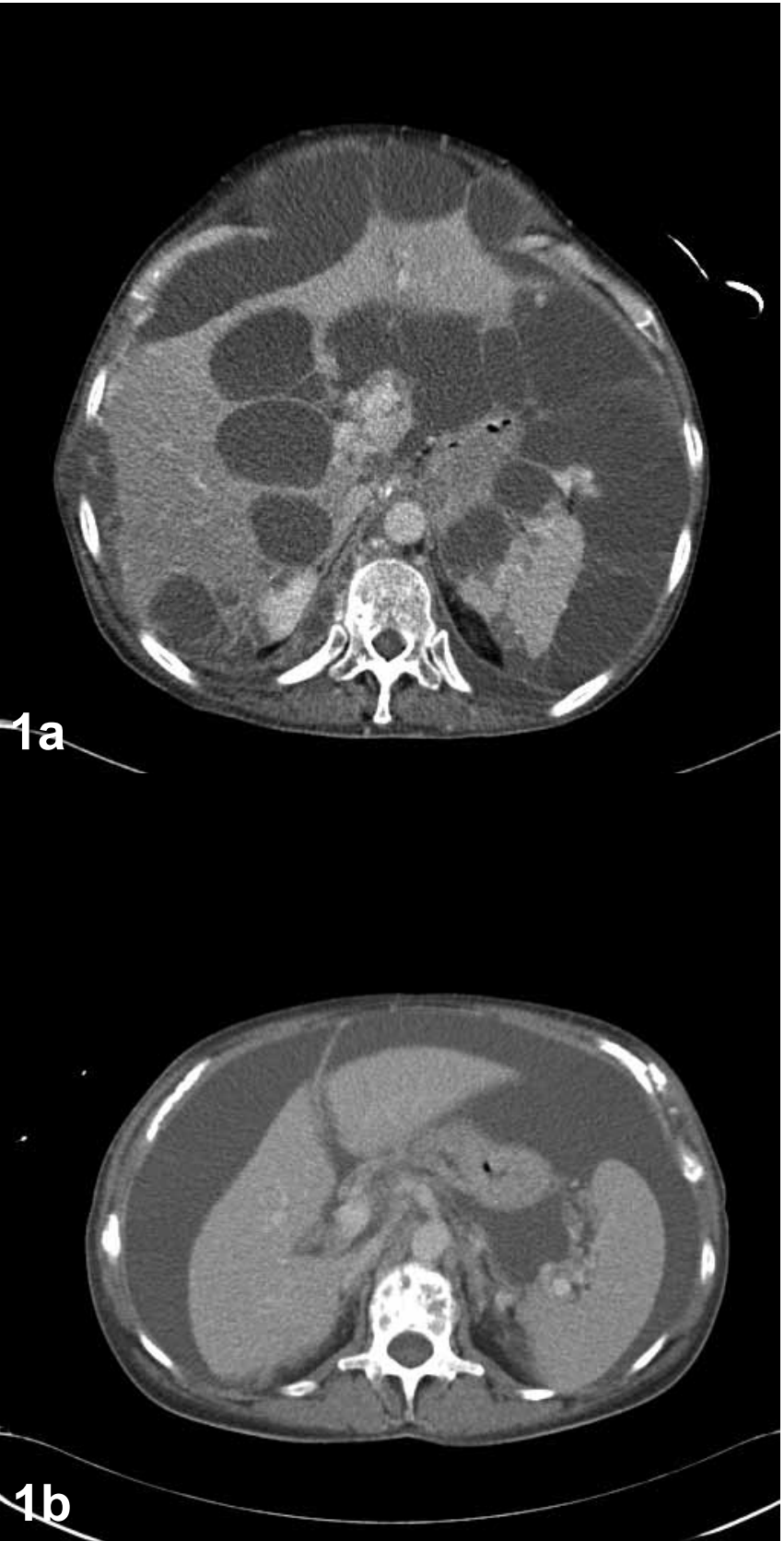

Fig. 1. Multiple complex cystic masses of fat density in the peritoneum (1a), and characteristic scalloping of the liver and spleen margins ( $1 b$ ). 


\section{REVIEW ARTICLE}

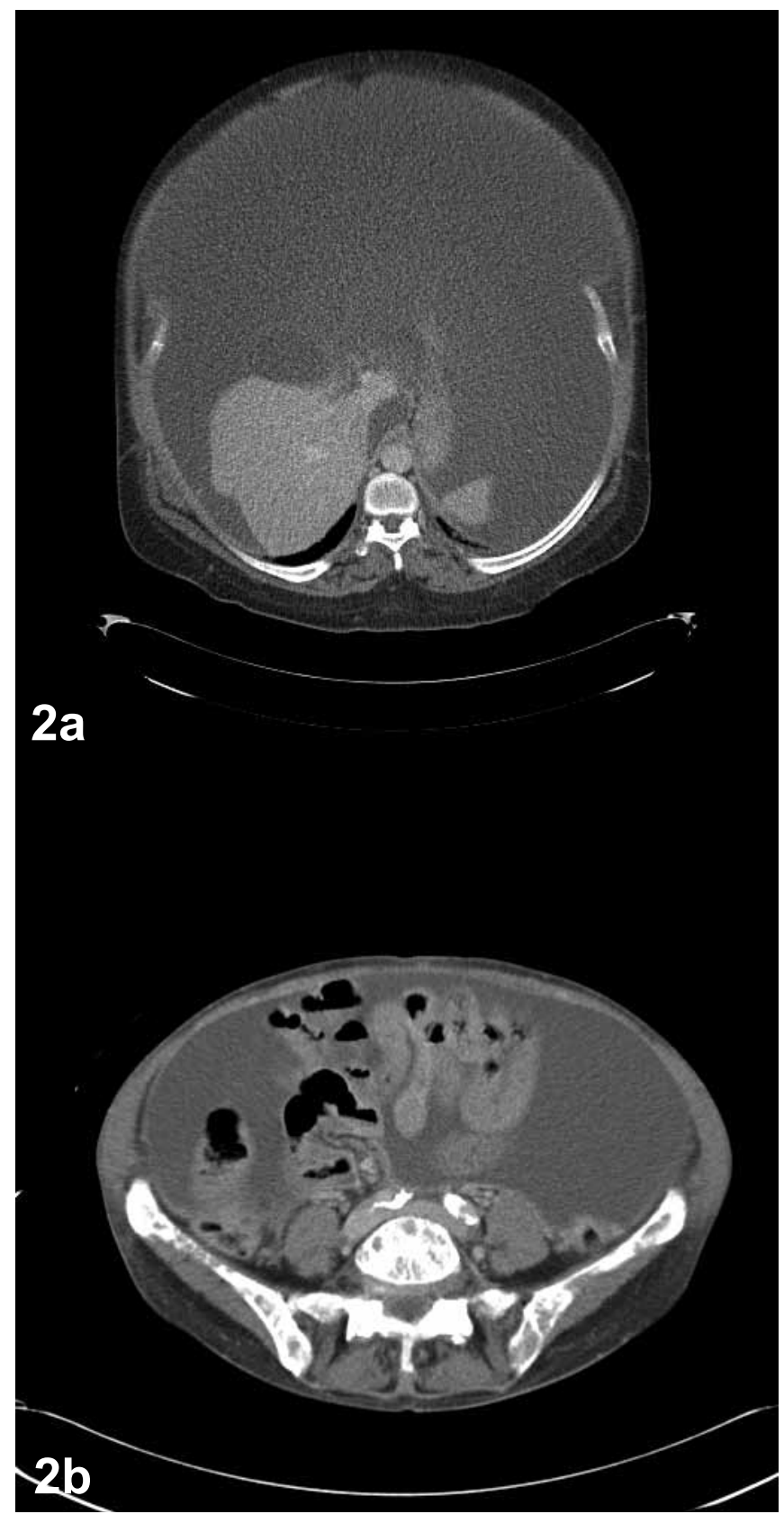

Figs $2 a$ and $2 b$. Compression of varying degrees of the visceral organs and structures.

stays localised to the abdomen; peritoneal mucinous carcinomatosis (PMCA) which has a metastatic potential; and an intermediate subtype (PMCA-I) which has invasive and metastatic potential with the possibilities of liver, lung and lymph node metastases. ${ }^{4}$

Recent research indicates that the PMP clinical symptoms may be caused by an overwhelming production in mucin 2 protein (MUC2) secreting cells, as well as the fact that the excessively produced mucin has no place to drain; this raises the possibility of MUC2-targeted therapy. $^{5}$

\section{Imaging findings}

Computed tomography (CT) is the modality of choice as it allows relatively accurate localisation and quantification of PMP. $^{6}$ CT

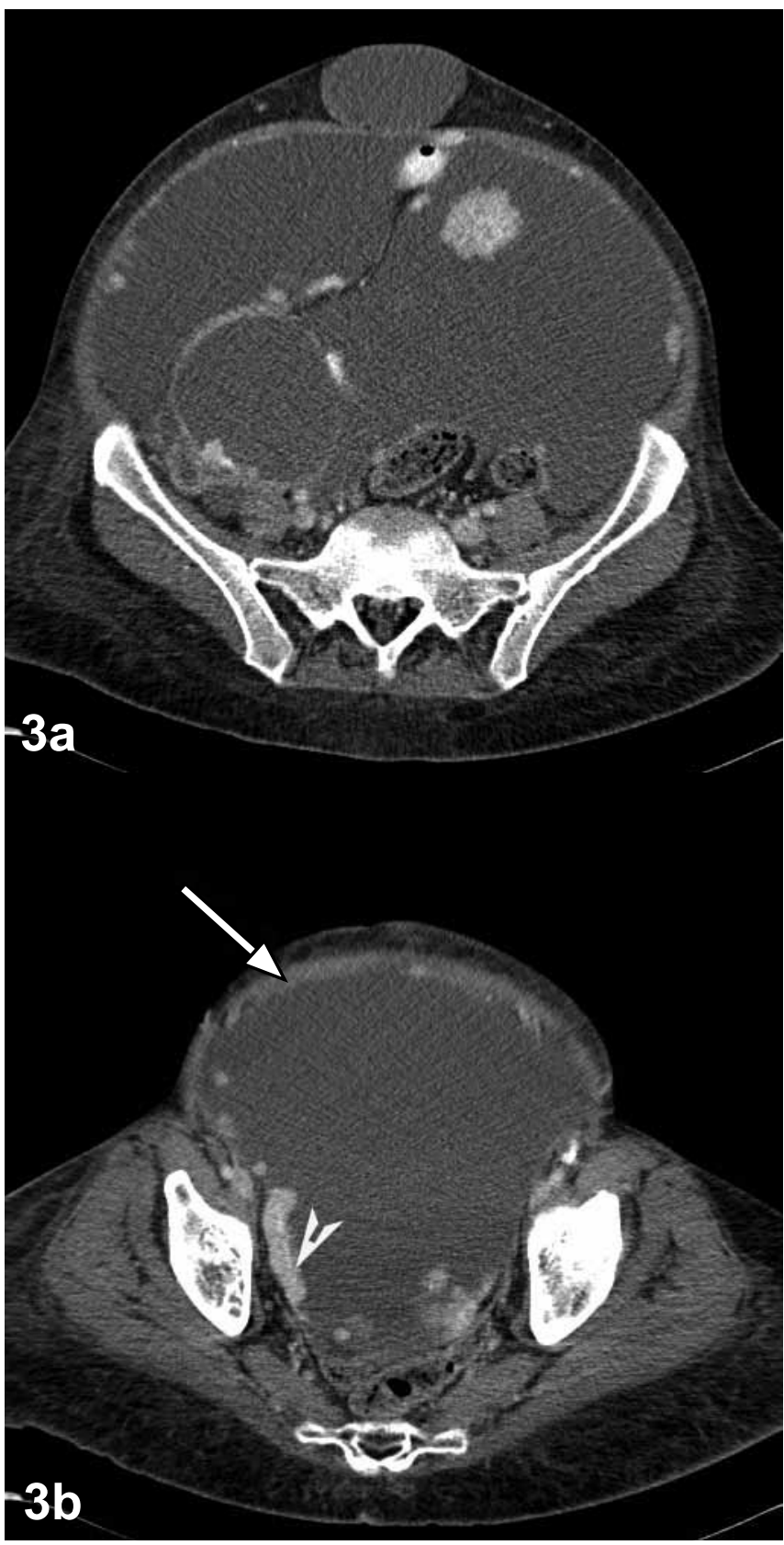

Figs $3 a$ and $3 b$. Omental thickening (arrow), omental (omental cake) and peritoneal implants (arrow head), and septated 'pseudo' ascites.

characteristics of PMP include: multiple complex cystic masses of low attenuation in the peritoneum, which may have rims of calcifications; characteristic scalloping of the liver (and occasionally splenic) margins; omental thickening, omental 'cake' and peritoneal implants; septated 'pseudo' ascites and varying degrees of compression of the visceral organs and structures (Figs $1-3$ ) ${ }^{3-9}$ Ultrasound often reveals gross, non-mobile ascites with septations and echogenicity. When correlated clinically, these radiologic features are highly specific for PMP. In patients with incidental findings of PMP, plain films of the abdomen may reveal abdominal calcific plaques, ascites and poorly-defined soft-tissue masses. These plain-film findings should be followed by CT studies. ${ }^{6} \mathrm{CT}$ can also be used to follow-up and re-evaluate patients. 


\section{REVIEW ARTICLE}

\section{Treatment}

Although therapeutic paracentesis is not possible because of the nature of the mucin, enough material can be removed by ultrasound guidance for diagnostic evaluation, if the diagnosis is not certain. This is rarely required as the history, examination, and imaging studies often lead to the diagnosis.

Currently, cytoreductive surgery combined with peri-operative intraperitoneal chemotherapy is the standard treatment for patients with peritoneal spread of primary appendiceal tumours. This is achieved by combining peritonectomy procedures with peri-operative intraperitoneal chemotherapy. ${ }^{8}$ PMP of ovarian origin is usually treated surgically by hysterectomy, bilateral salpingo-oophorectomy, prophylactic appendectomy, and general debulking of the mucin. ${ }^{3}$

\section{Differential diagnosis}

The differential diagnosis includes primary or secondary peritoneal tumours, which may also present with scalloped liver margins and septated ascites. ${ }^{10}$ Pancreatitis with ascites and pancreatic pseudocysts may also form part of the differential diagnosis, although the presence of pancreatic abnormalities may allude to this diagnosis. Infective causes such as pyogenic peritonitis, widespread echinococcal disease and TB peritonitis, may be considered but the clinical presentation of these patients as well as the presence of other imaging findings such as liver abscesses or micro-abscesses (in the case of TB) may also be present.

1. Devang, B, Allen C, Sean C. Pseudomyxoma peritonei. App Radiol 2005;34:6-8.

2. Dahnert WF. Radiology Review Manual, 5th ed. Philadelphia: Lippincott Williams and Wilkins, 2007.

3. Harshen R, Jyothirmayi R, Mithal N. Pseudomyxoma peritonei. Clin Oncol 2003;15:73-77.

4. Chen CF, Huang CJ, Kang WY, Hsieh JS.Experience with adjuvant chemotherapy for pseudomyxoma peritonei secondary to mucinous adenocarcinoma of the appendix with oxaliplatin/fluorouracil/leucovorin (FOLFOX4) World Journal of Surgical Oncology 2008;6:118.

5. O'Connell JT, Tomlinson JS, Roberts AA, McGonigle KF, Barsky SH. Pseudomyxoma peritonei is a disease of MUC2-expressing goblet cells. Am J Pathol 2002;161:551-564.

6. Lee H, Agha F, Weatherbee L, Boland C.Pseudomyxoma peritonei. Radiologic features. J Clin Gastroenterol 1986;8:312-316.

7. Pickhardt PJ, Levy AD, Rohrmann CA Jr, Kende AI. Primary neoplasms of the appendix: Radiologic spectrum of disease with pathologic correlation. RadioGraphics 2003;23:645-662.

8. Sugarbaker P. Cytoreductive surgery and peri-operative intraperitoneal chemotherapy as a curative approach to pseudomyxoma peritonei syndrome. Eur J Surg Oncol 2001;27:239-243.

9. Weissleder R, Wittenberg J, Mukesh GH, John WC. Primer of Diagnostic Imaging. Philadelphia: Mosby Elsevier, 2007.

10. Gupta S, Gupta RK, Gujral RB, Saxena DAR, Tandon P. Peritoneal mesothelioma simulating pseudomyxoma peritonei on CT. Gastrointestinal Radiology 1992;17:129-131. SonographyGastrointest Radiol 17:129-131 (19 